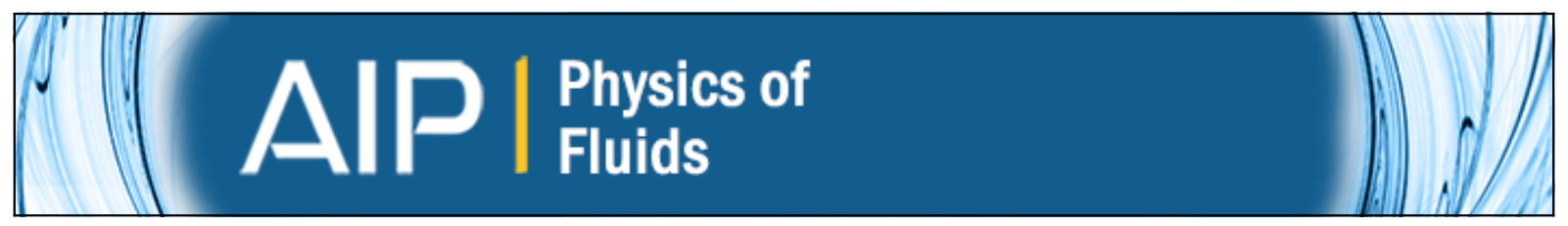

\title{
Spontaneous penetration of a non-wetting drop into an exposed pore
}

Pengtao Yue and Yuriko Renardy

Citation: Physics of Fluids (1994-present) 25, 052104 (2013); doi: 10.1063/1.4804957

View online: http://dx.doi.org/10.1063/1.4804957

View Table of Contents: http://scitation.aip.org/content/aip/journal/pof2/25/5?ver=pdfcov

Published by the AIP Publishing

Copyright by the American

Institute of Physics.

Spontaneous penetration of a

non-wetting drop into an exposed

pore. Yue, Pengtao and Renardy,

Yuriko, Physics of Fluids (1994-

present), 25, 052104 (2013),

DOI $:$ http: //

dx.doi.org/10.1063/1.4804957

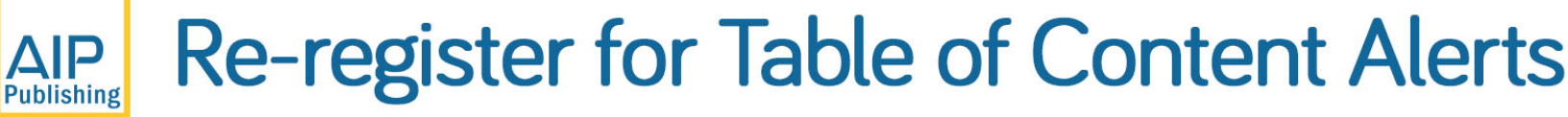

\section{Create a profile. 四 Sign up today!}




\title{
Spontaneous penetration of a non-wetting drop into an exposed pore
}

\author{
Pengtao Yue ${ }^{\mathrm{a})}$ and Yuriko Renardy ${ }^{\mathrm{b}}$ ) \\ Department of Mathematics, Virginia Tech, Blacksburg, Virginia 24061-0123, USA
}

(Received 16 October 2012; accepted 30 April 2013; published online 21 May 2013)

\begin{abstract}
We consider the penetration process of a liquid drop approaching an exposed pore along the axis of symmetry, which is intended to model the penetration of non-wetting drops into a porous medium. Inertia and gravity are neglected at the current stage. In addition to the penetration into a capillary tube in the literature, the drop may spread on the outer surface of the porous medium. Based on the mechanical equilibrium states, we find the critical drop radius, below which the drop penetration is spontaneous. We further identify five penetration regimes based on the drop radius and the static contact angle, all of which are exemplified by phase-field simulations. The free energy as a function of penetration depth reveals only two stable equilibrium states: the drop either enters the pore completely (maximum penetration) or stays at the pore inlet (zero penetration). For a non-penetrating drop radius, the free energy has a local maximum which constitutes an energy barrier that prevents spontaneous penetration. Finally, we modify the Lucas-Washburn equation to describe the dynamic process of penetration. Due to the neglect of dissipation from moving contact lines and entry flow, the modified Lucas-Washburn equation greatly overestimates the penetration rate, especially at the initial stage. @ 2013 AIP Publishing LLC. [http://dx.doi.org/10.1063/1.4804957]
\end{abstract}

\section{INTRODUCTION}

The wetting of porous media has been an active research topic for many years due to its importance in a wide range of applications such as oil recovery, ${ }^{1}$ textile technology, ${ }^{2}$ ink jet printing, ${ }^{3}$ and fuel cell technology. ${ }^{4}$ For such a complex problem, it is crucial to have a good understanding of wetting in a single pore as a first step. The most prominent work is perhaps that by Lucas ${ }^{5}$ and Washburn, ${ }^{6}$ who first rigorously analyzed the capillary rise in cylindrical tubes. Their analysis assumes a fully developed Poiseuille flow and neglects inertia. The kinetics of penetration also depends on many other factors such as inertia, dynamic contact angle, fluid rheology, and even shape variations of the channel, which are addressed in later work. ${ }^{7-10}$

Up to now, most work on liquid penetration in capillaries have focused on the capillary tubes connected to infinite liquid reservoirs. ${ }^{11-13}$ This is a good approximation when a large drop is deposited on a porous medium. However, for small drops, the size effect has to be considered especially when it is comparable with the capillary size. The curved drop surface may generate a capillary pressure that promotes drop penetration. Marmur ${ }^{14}$ is the first to incorporate this effect into analysis. He found that a non-wetting drop may still penetrate the capillary tube if its initial radius satisfies

$$
R_{0}<\frac{r_{0}}{-\cos \theta_{S}},
$$

where $r_{0}$ is the inner radius of the capillary tube, and $\theta_{S}>90^{\circ}$ is the static contact angle. For a wetting drop $\left(\theta_{S}<90^{\circ}\right)$, the penetration rate is higher than that for an infinite reservoir. Marmur also

\footnotetext{
a)Electronic mail: ptyue@math.vt.edu

b)Electronic mail: renardy@math.vt.edu
} 
proposed a radial capillary model which uses two parallel plates, instead of a cylindrical capillary, to model drop penetration in a thin porous medium. ${ }^{15}$ Denseuk et al. extended Marmur's work and modeled the porous medium as a solid body with cylindrical pores uniformly distributed over the surface. ${ }^{16}$ Hapgood et al. further considered the effect of macrovoids in the computation of penetration times in porous media. ${ }^{17}$ However, the Laplace pressure inside the drop was neglected in the latter two works.

Recently, the research on drop penetration into capillaries is brought into attention again due to the development of carbon nanotubes. ${ }^{18}$ Carbon nanotubes may be used as capillaries to uptake and release nanodroplets. ${ }^{19}$ Capillarity also plays an important role in the withdrawal of metal catalyst particles during the growth of nanotubes and nanofibers by the chemical vapor deposition technique. ${ }^{20}$ The most relevant work is that by Schebarchov and Hendy, ${ }^{19,21,22}$ who applied Marmur's analysis to nanotubes and conducted molecular dynamics simulations. Slip at the tube wall is the major difference compared with the problem at a macroscopic length scale. By studying the evolution of surface energy, these authors identified an energy barrier for the uptake of drops with radii larger than that predicted by Eq. (1). At the nanometer scale, this energy barrier may be overcome by the thermal fluctuations, which is verified by molecular dynamics simulations. The experimental evidence of drop uptake by non-wetting capillaries can be found in Ref. 23, although the tube diameter is actually at the order of a millimeter.

In all the previous theoretical analyses, it has been assumed that the contact line where the surface of the protruding drop intersects the solid surface is pinned at the tube inlet. This is valid for capillary tubes with relatively thin tube walls. However, for a porous media, this is no longer true as the drop may spread on the outer surface of the media. In this case, it is more practical to consider a pore bored into a solid surface, which allows the contact line to spread out, as shown in Fig. 3. In this work, we study such an exposed pore both theoretically and numerically. We first provide theoretical analysis on the drop penetration process, which is supplemented by phase-field simulations. Then we discuss the energy barrier for large drops. Finally, we study the transient behavior of penetration and show that the Lucas-Washburn equation is not sufficient to describe the penetration dynamics.

\section{PHASE-FIELD FORMULATIONS}

The numerical simulation of drop penetration requires the proper treatment of contact line dynamics. We adopt the phase-field method, which is also known as the diffuse-interface method. This method utilizes the Cahn-Hilliard diffusion to regularize the stress singularity at moving contact lines and has gained popularity in the simulation of dynamic wetting. ${ }^{24-26}$ Recently, Mehrabian et $a l .{ }^{27}$ used the phase-field method to study the wicking flow through complex microchannels, which tackles another aspect of liquid penetration in porous media. In the following, we briefly describe the governing equations and numerical methods.

We consider the penetration of a Newtonian drop into a pore, as shown in Fig. 3. A phase-field variable $\phi$, which assumes 1 in the liquid and -1 in the gas, is used to identify the diffuse interface. For simplicity, we neglect inertia and gravity, which is a valid approximation for small length scales. The governing equations consist of the Cahn-Hilliard equation for the interface, the momentum equation, and the incompressible continuity equation:

$$
\begin{gathered}
\frac{\partial \phi}{\partial t}+\boldsymbol{v} \cdot \phi=\gamma \nabla^{2} G, \\
\nabla \cdot(-p \boldsymbol{I}+\boldsymbol{\tau})+G \nabla \phi=0, \\
\nabla \cdot \boldsymbol{v}=0,
\end{gathered}
$$

where

$$
G=\lambda\left[-\nabla^{2} \phi+\frac{\phi\left(\phi^{2}-1\right)}{\epsilon^{2}}\right]
$$




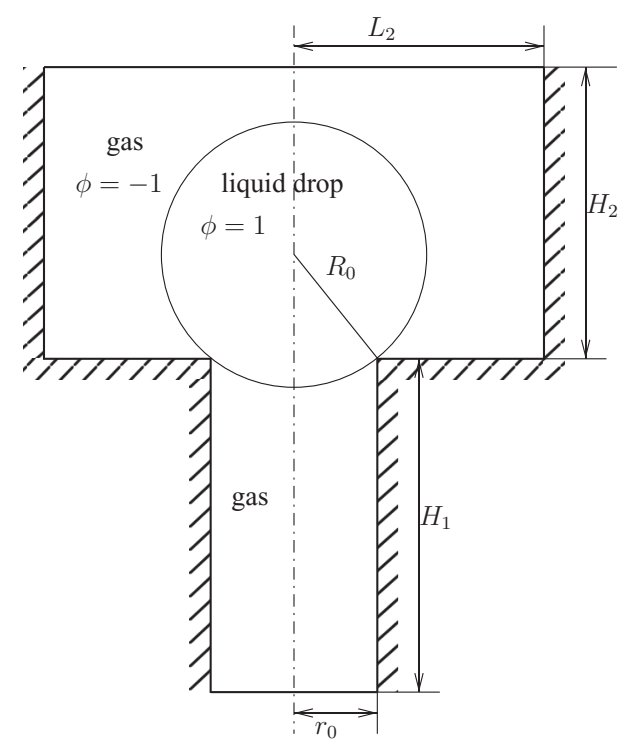

FIG. 1. Schematic of computational domain.

is the chemcial potential and

$$
\boldsymbol{\tau}=\bar{\eta}\left(\nabla \boldsymbol{v}+(\nabla \boldsymbol{v})^{T}\right)
$$

is the viscous stress. Here $\gamma$ is the Cahn-Hilliard mobility paramter, $\epsilon$ is a parameter which is proportional to the interfacial thickness, $\lambda$ is the mixing energy density which is related to the interfacial tension $\sigma$ by $\sigma=\frac{2 \sqrt{2}}{3} \frac{\lambda}{\epsilon} \cdot \bar{\eta}$ is the mixture viscosity defined as

$$
\bar{\eta}=\frac{1+\phi}{2} \eta+\frac{1-\phi}{2} \eta_{g}
$$

where $\eta$ and $\eta_{g}$ are the viscosities of the liquid and the gas, respectively.

The computational domain is shown in Fig. 1. We consider the flow to be axisymmetric and only compute one half of the median plane. $r_{0}$ is the inner radius of the pore and $R_{0}$ is the radius of the initial drop. We choose $H_{1}=12 r_{0}, H_{2}=6 r_{0}$, and $L_{2}=4 r_{0}$ so that the drops computed in this paper $\left(R_{0} \leq 2.5 r_{0}\right)$ do not touch boundaries other than the pore wall and the horizontal wall. Initially, the drop is spherical and barely touches the pore inlet. On the solid walls (the hatched boundaries), we impose the no-slip, no-flux, and contact angle conditions:

$$
\begin{gathered}
\boldsymbol{v}=0, \\
\boldsymbol{n} \cdot \nabla G=0, \\
\lambda \boldsymbol{n} \cdot \nabla \boldsymbol{\phi}+f_{w}^{\prime}(\phi)=0,
\end{gathered}
$$

where $\boldsymbol{n}$ is the outward pointing normal to the boundary and $f_{w}$ is the wall energy which is related to the static contact angle $\theta_{S}$. On a solid substrate as shown in Fig. $2, f_{w}$ is given by

$$
f_{w}(\phi)=-\sigma \cos \theta_{S} \frac{\phi\left(3-\phi^{2}\right)}{4}+\frac{\sigma_{w 1}+\sigma_{w 2}}{2},
$$

where $\sigma_{w 1}$ and $\sigma_{w 2}$ are the liquid-solid and gas-solid interfacial tensions. Note that the wall energy relaxation is not considered in Eq. (10), and therefore the microscopic contact angle is fixed at $\theta_{S}{ }^{28}$ At the channel openings, we impose the zero normal stress condition

$$
\boldsymbol{n} \cdot(-p \boldsymbol{I}+\boldsymbol{\tau})=0,
$$

so that the drop motion is completely driven by capillarity. 


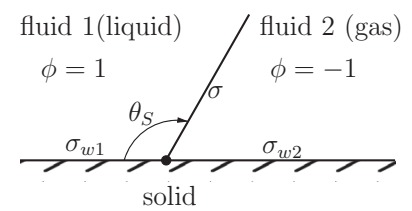

FIG. 2. Schematic of a static contact line.

In this paper, we use pore radius $r_{0}$, liquid viscosity $\eta$, and interfacial tension $\sigma$ to nondimensionalize all the quantities. Consequently, time is scaled by $\eta r_{0} / \sigma$ and energy is scaled by $\sigma r_{0}^{2}$. In the following, all the numbers are dimensionless unless otherwise indicated. In all computations, we use $r_{0}=1, \eta=1, \eta_{g}=0.02 \ll \eta, \sigma=1, \gamma=1 \times 10^{-4}$, and $\epsilon=0.01 . R_{0}$ and $\theta_{S}$ are varied case by case. According to Yue et al.,$^{29}$ the diffusion length is $l_{D}=\sqrt{\gamma \sqrt{\eta \eta_{g}}}=3.761 \times 10^{-3}$, which corresponds to a slip length $l_{s} \approx 2.5 l_{D}=9.401 \times 10^{-3}$. The cell size of the computational mesh is 0.005 at the interface, 0.1 inside the drop, and 0.2 in the air. This sufficiently resolves the diffuse interface and the slip length.

The governing equations (Eqs. (2)-(4)) together with the boundary conditions (Eqs. (8)-(12)) are solved by a finite-element method on an adaptive triangular mesh. All the time-dependent equations are integrated using a second-order accurate, fully implicit time-marching scheme. Numerical details and code validation can be found in Ref. 30. More details of the phase-field method and its application in dynamic wetting can be found in Refs. 28, 29, 31, and 32.

\section{DISTINCT REGIMES OF DROP PENETRATION}

At static equilibrium, as shown in Fig. 2, $\theta_{S}$ can be obtained from Young's relation

$$
\sigma \cos \theta_{S}+\sigma_{w 1}-\sigma_{w 2}=0
$$

which is a force balance at the contact line. If the contact angle $\theta$ deviates from $\theta_{S}$, an unbalanced Young's force

$$
f_{Y}=\sigma_{w 2}-\sigma_{w 1}-\sigma \cos \theta=\sigma\left(\cos \theta_{S}-\cos \theta\right)
$$

acts on the contact line, and consequently the contact line moves leftward if $\theta<\theta_{S}\left(f_{Y}<0\right)$ or rightward if $\theta>\theta_{S}\left(f_{Y}>0\right)$. In the following, we only consider the non-wetting case, i.e., $\theta_{S}>\pi / 2$.

When a spherical drop barely touches the pore inlet, two contact angles $\theta_{1}=\arcsin \left(1 / R_{0}\right)+\pi / 2$ and $\theta_{2}=3 \pi / 2-\theta_{1}$ can be defined as shown in Fig. 3(a). For convenience we define the contact line on the outer horizontal surface as $B$ and the contact line on the pore inner surface as $A$. It is obvious that $A$ will move into the pore if $\theta_{1}>\theta_{S}$ (Figs. 3(c)-3(e)) and stays at $C$ otherwise (Figs. 3(a) and 3(b)). Similarly, $B$ will spread out on the outer surface if $\theta_{2}>\theta_{S}$ (Figs. 3(b) and 3(c)) and stays at $C$ otherwise (Figs. 3(a), 3(d), and 3(e)).

The equilibrium states of drop penetration can be obtained from three balance conditions: Young's relation at the contact line, Young-Laplace equation $\Delta p=2 \sigma / R$ ( $R$ being the radius of curvature) across the interface, and uniform pressure inside the quiescent drop. The first condition predicts that contact angle is fixed at $\theta_{S}$, except at the corner point $C$ where the contact angle is not well defined. The second and the third conditions together require that all the fluid interfaces are spherical and have the same curvature. Considering all these conditions, we can come up with five possibilities of the equilibrium states, as shown in Fig. 3. In later sections, we will see that the equilibrium states in (c) and (d) are unstable.

Based on the angles in Fig. 3(a), we can define two critical radii:

$$
R_{p}=\frac{r_{0}}{-\cos \theta_{S}}
$$

which corresponds to $\theta_{1}=\theta_{S}$ and

$$
R_{s}=\frac{r_{0}}{\sin \theta_{S}}
$$




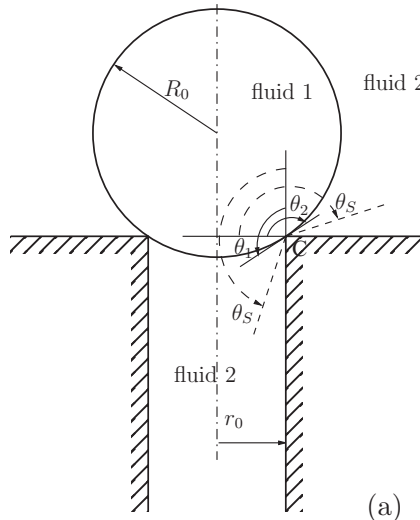

(a)

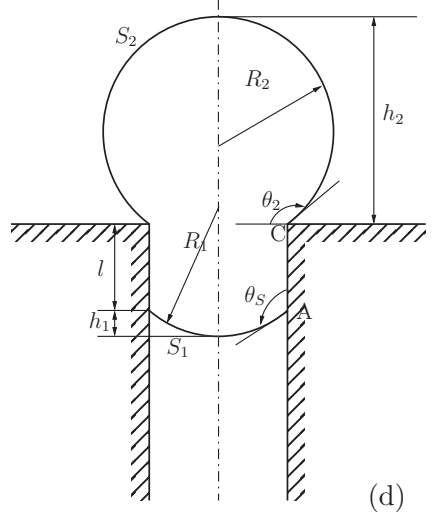

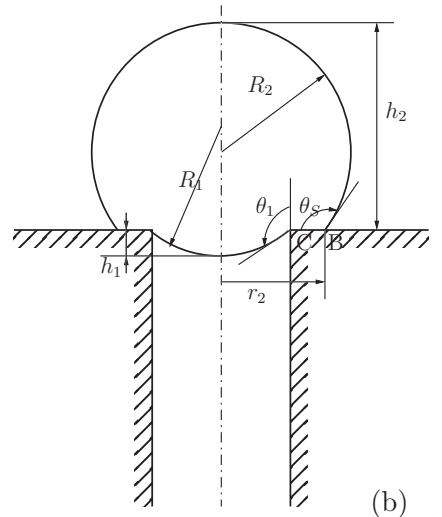

(b)

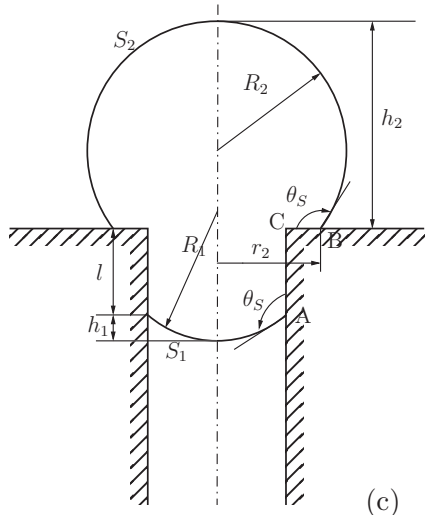

(c)

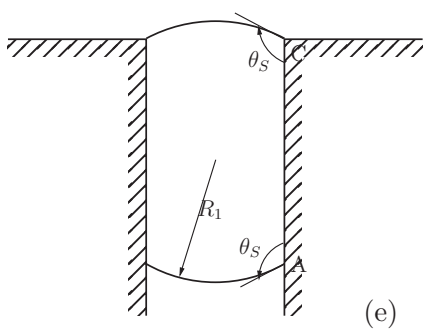

FIG. 3. Possible equilibrium configurations when a drop enters a pore. (a) spherical drop; (b) zero penetration with surface spreading; (c) partial penetration with surface spreading; (d) partial penetration with no surface spreading; (e) complete penetration. $r_{0}$ is the pore radius, $R_{0}$ is the initial drop radius, and $l$ is the penetration depth. Equilibration requires $R_{1}=R_{2}$. $\pi / 2<\theta_{1} \leq \theta_{S}$ in (b) and $\pi / 2<\theta_{2} \leq \theta_{S}$ in (d).

which corresponds to $\theta_{2}=\theta_{S}$. Contact line $A$ moves into the pore when $R_{0}<R_{p}$ and $B$ spreads out on outer surface when $R_{0}>R_{s}$. Note that, $R_{p}$ is also Marmur's critical drop radius for spontaneous penetration when there is no spreading on the outer surface. ${ }^{14} \mathrm{~A}$ third critical radius for the drop with surface spreading, denoted by $R_{c}$, can be determined from the boundary between Figs. 3(b) and 3(c). We consider Fig. 3(b) with $\theta_{1}=\theta_{S}$. Contact line $A$ enters the pore if $R_{0}<R_{c}\left(\theta_{1}>\theta_{S}\right)$ or remains at the corner if $R_{0}>R_{c}\left(\theta_{1}<\theta_{S}\right)$. The volume of the spherical cap inside the pore is

$$
V_{1}=\frac{\pi}{6} h_{1}\left(3 r_{0}^{2}+h_{1}^{2}\right)
$$

and the volume of the spherical cap outside is

$$
V_{2}=\frac{\pi}{6} h_{2}\left(3 r_{2}^{2}+h_{2}^{2}\right)
$$

where $h_{1}, h_{2}$, and $r_{2}$ are indicated in Fig. 3(c). Considering the constraints $\theta_{1}=\theta_{S}$ and $R_{1}=R_{2}$, we can easily get $R_{1}=R_{2}=\frac{r_{0}}{-\cos \theta_{S}}, h_{1}=R_{1}\left[1-\cos \left(\theta_{S}-\pi / 2\right)\right]=r_{0}\left(1-\sin \theta_{S}\right) /\left(-\cos \theta_{S}\right)$, $h_{2}=R_{2}\left[1+\sin \left(\theta_{S}-\pi / 2\right)\right]=r_{0}\left(1-\cos \theta_{S}\right) /\left(-\cos \theta_{S}\right)$, and $r_{2}=-r_{0} \tan \theta_{S}$. Note that this analysis is only valid for $\theta_{S} \in[\pi / 2,3 \pi / 4]$ in order to satisfy the geometric requirement $r_{2} \geq r_{0}$. Finally, from volume conservation $\frac{4 \pi}{3} R_{c}^{3}=V_{1}+V 2$, we get

$$
R_{c}=\frac{r_{0}}{-\cos \theta_{S}} f\left(\theta_{S}\right)
$$




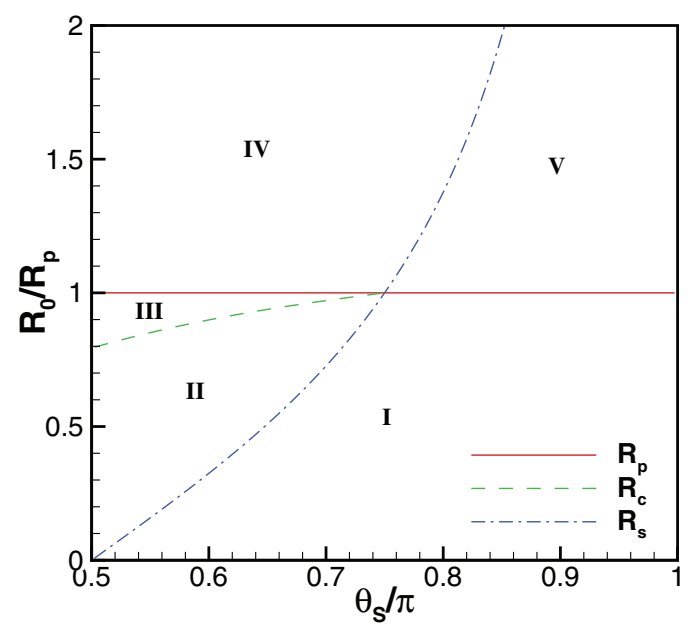

FIG. 4. Penetration regimes.

where

$$
f\left(\theta_{S}\right)=\sqrt[3]{1-\frac{9 \cos \theta_{S}-\cos \left(3 \theta_{S}\right)+9 \sin \theta_{S}+\sin \left(3 \theta_{S}\right)}{16}}
$$

is an increasing function on $\theta_{S} \in[\pi / 2,3 \pi / 4]$. In particular, $f(\pi / 2)=\sqrt[3]{1 / 2}$ and $f(3 \pi / 4)=1$.

These three radii divide the $\theta_{S}-R_{0}$ plane into 5 regimes, as shown in Fig. 4. The $R_{c}$ and $R_{S}$ curves are actually $f\left(\theta_{S}\right)$ and $\cot \left(\theta_{S}\right)$, respectively. In Regime I, the drop penetrates into the pore without any spreading on the outer solid surface; the final state is Fig. 3(e). In Regime II, the drop spreads on the outer solid surface initially, and eventually penetrates into the pore; the final state is still Fig. 3(e). In Regime III, the drop spreads on the outer solid surface initially; the initial transient determines whether the drop penetrates into the pore or not; the final state is Fig. 3(b) or 3(e). In Regime IV, the drop spreads on the outer solid surface without any penetration; the final state is Fig. 3(b). In Regime V, the drop remains spherical and the final state is Fig. 3(a). In the following we will discuss these regimes in details.

\section{A. $\theta s \in(\pi / 2,3 \pi / 4)$}

Four regimes are involved for this $\theta_{S}$. We run phase-field simulations with $\theta_{S}=2 \pi / 3$, which corresponds to $R_{p}=2 r_{0}, R_{c}=1.8994 r_{0}$, and $R_{s}=\frac{2}{\sqrt{3}} r_{0}=1.1547 r_{0}$ to illustrate the drop penetration process.

\section{Regime $I, R_{0} \in\left(r_{0}, R_{s}\right)$}

The penetration process with $R_{0}=1.1$ is shown in Fig. 5. The $\phi=0$ contours are plotted to indicate the interface location. A close view at the contact line is shown in Fig. 6. Initially, the diffuse interface of the drop just cuts through the corner of the solid boundary, as shown in Fig. 6(a). Because $\theta_{1}=155.4^{\circ}>\theta_{S}$ and $\theta_{2}=114.6^{\circ}<\theta_{S}$, contact line $A$ will move into the pore while the contact line $B$ will stay at the corner. Once contact line $A$ leaves the corner, the microscopic contact angle remains fixed at $\theta_{S}$, as shown in Fig. 6(b). Due to viscous bending at the contact line, the interface inclination angle quickly relaxes from $\theta_{S}$ to the apparent contact angle $\theta_{A}$, which is sometimes referred to as the dynamic contact angle. There are different ways to measure $\theta_{A}$ and the value is not unique. For the capillary rise problem, $\theta_{A}$ is usually defined as the angle made by the wall and the circular fit to the meniscus. ${ }^{33}$ Shortly after $t=0$, as shown in Fig. 6(b), the circular fit to the meniscus has a radius $R_{1}=1.130$, from which we obtain $\theta_{A}=146.67^{\circ}>\theta_{S} . \theta_{A}$ approaches $\theta_{S}$ as the contact line slows down. In Fig. $6(\mathrm{c}), \theta_{A}$ reaches $120.2^{\circ}$ which is already very close to $\theta_{S}$. 

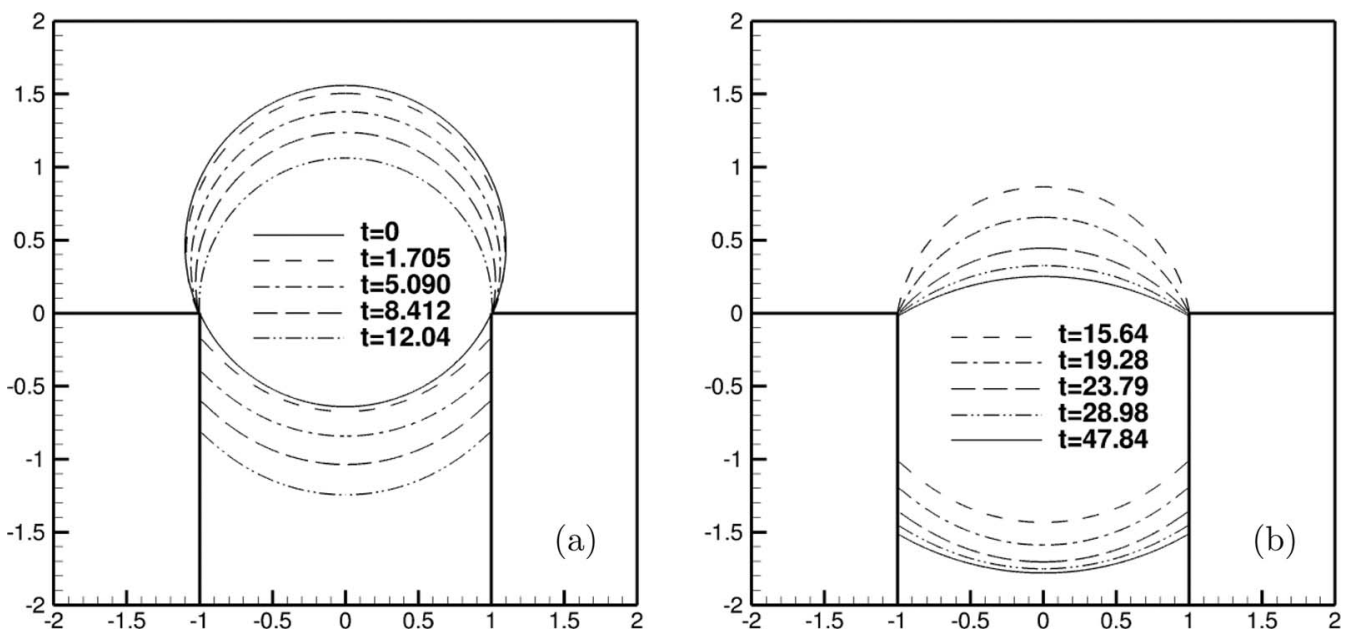

FIG. 5. Drop penetration with $\theta_{S} \in(\pi / 2,3 \pi / 4)$ and $R_{0} \in\left(r_{0}, R_{S}\right) . \theta_{S}=2 \pi / 3, R_{0}=1.1$.

Eventually, the complete penetration is achieved. Note that it takes infinite time to achieve the exact final equilibrium (cf. Fig. 3(e)) as the system is overdamped.

\section{Regime II, $R_{0} \in\left(R_{s}, R_{c}\right)$}

We choose $R_{0}=1.5$ for this case. The evolution of the drop interface is shown in Fig. 7 and typical snapshots of the flow field are shown in Fig. 8. Initially, we have $\theta_{1}=131.8^{\circ}>\theta_{S}$ and $\theta_{2}$ $=138.2^{\circ}>\theta_{S}$. From $t=0$ to $t=21.69$ in Fig. 7(a), contact line $A$ moves into the pore and $B$ spreads out along the outer surface, which is also borne out by the streamlines in Fig. 8(a). At $t=21.69$, the apparent contact angle $\theta_{A}$ is measured to be $120.0^{\circ}=\theta_{S}$; therefore contact line $B$ stops. If we assume that there is no flow, then the radius of the protruding drop $R_{2}$ is always smaller than that of the meniscus $R_{1}=R_{p}$ because $R_{0}<R_{c}$, let alone the extra drop volume consumed by the non-zero penetration depth $l$. Note that the drop surface is not exactly spherical if flow exists, although it is still a good approximation. Therefore, the Laplace pressure inside the drop is higher at the top and is lower at the bottom, which tends to push the drop into the pore, as shown in Fig. 8(b). As a result, the protruding drop retracts and the meniscus moves downward, as shown in Fig. 7(b). At $t=136.8$, contact line $B$ reaches the corner and stays pinned thereafter. The protruding drop keeps shrinking until its radius passes the minimum $r_{0}$ and then increases to $R_{2}=R_{1}=R_{p}$, as shown in Fig. 7(c).

\section{Regime III, $R_{0} \in\left(R_{c}, R_{p}\right)$}

Different from Regime II, the drop in this regime is not guaranteed to enter the pore. The results for $R_{0}=1.95$ are shown in Figs. 9 and 10. Initially, $\theta_{1}=120.9^{\circ}>\theta_{s}, \theta_{2}=149.1^{\circ}>\theta_{s}$. Contact
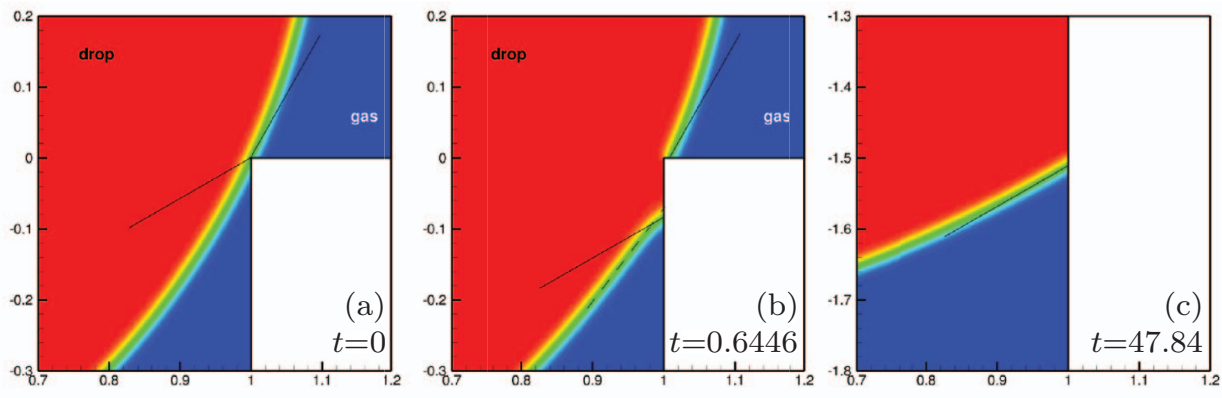

FIG. 6. (a)-(c) $\phi$ contours near the contact line. $\theta_{S}=2 \pi / 3, R_{0}=1$.1. The solid line indicates $\theta_{S}$ and the dashed line indicates the apparent contact angle $\theta_{A}$. 

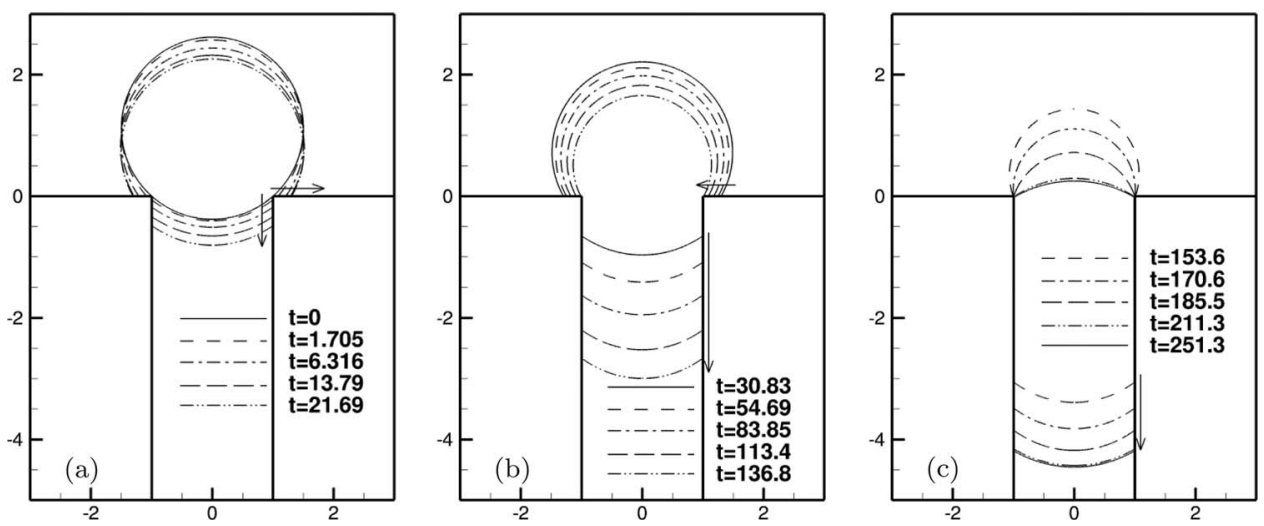

FIG. 7. (a)-(c) Drop penetration with $\theta_{S} \in(\pi / 2,3 \pi / 4)$ and $R_{0} \in\left(R_{S}, R_{c}\right) . \theta_{S}=2 \pi / 3, R_{0}=1.5$. Arrows indicate the moving direction of the contact lines.

lines $A$ and $B$ both move away from the corner, which is the same as that in Regime II. But, because $R_{0}>R_{c}$, it is very possible to have $R_{2}>R_{1}$. As a result, the capillary pressure generated by the lower drop surface prevents the drop from entering the pore. We say possible here because whether $R_{2}>R_{1}$ also depends on the penetration depth $l$. If the penetration depth can exceed a critical value $l_{c}$ such that $R_{2}=R_{1}$, then it is still possible for the drop to enter the pore spontaneously. More discussions about this critical depth $l_{c}$ can be found in Sec. IV. In particular, we have $l_{c}=0.7498$ for $R_{0}=1.95$ according to Eq. (34). As can be seen from the inset of Fig. 9, at $t$ $=18.03, l$ maximizes at 0.06 , which is much less than $l_{c}$. Therefore we have $R_{2}<R_{1}$ and the meniscus begins to withdraw from the pore. The analysis is supported by the velocity field in Fig. 10. We do not plot pressure contours for this case because it is almost constant inside the drop. At $t=75.57$, the maximum velocity drops to $5 \times 10^{-5}$. The radii of curvature of the two drop surfaces are computed from circular fitting to be $R_{1}=2.047$ and $R_{2}=2.046$, which are almost in equilibrium. As $R_{1}>R_{p}$, the meniscus cannot sustain an apparent contact angle that is larger than $\theta_{S}$, so contact line $A$ has to move back to the corner.

This critical length decreases as $R_{0}$ approaches $R_{c}$. When $R_{0}=R_{c}=1.8994, l_{c}=0$ (cf. Eq. (34)) and the drop is guaranteed to penetrate the pore, as shown in Fig. 11. As $R_{0}=1.8994$ is close to 1.95 , the dynamics of initial drop spreading $(t \leq 73.71)$ is also close to that in Fig. 9. However, different from $R_{0}=1.95$, even a minute amount of penetration will lead to $R_{2}<R_{1}$. At $t$ $=73.71$, from circular fitting, the radii of the two drop surfaces are computed to be $R_{1}=2.005$ and $R_{2}=1.985$. This slight curvature difference drives a slow flow into the pore. Note that the flow is very weak and contact line $B$ barely moves between $t=73.71$ and 339.7. As the protruding drop
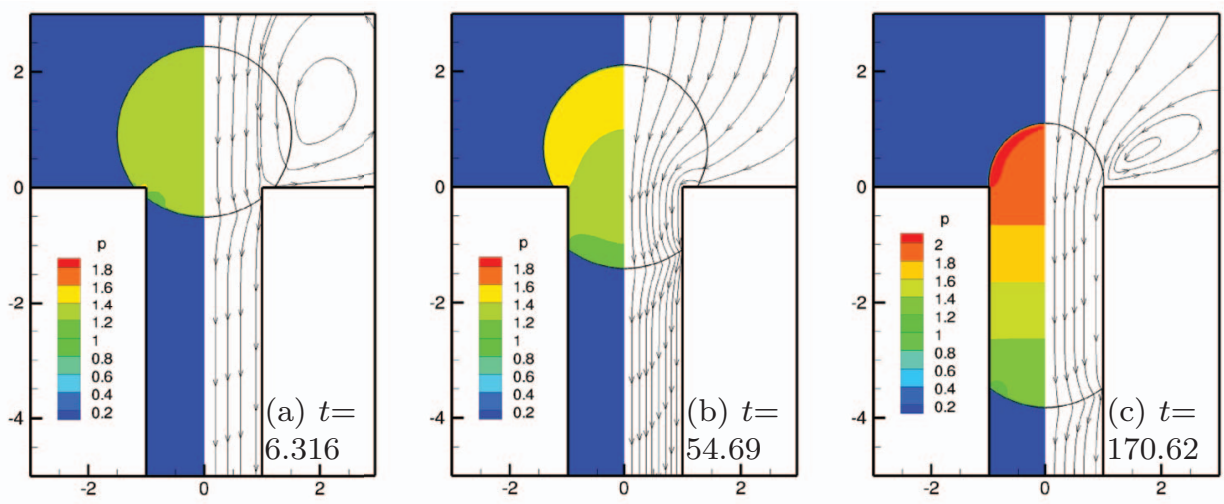

FIG. 8. (a)-(c) Pressure contours and streamlines. $\theta_{S}=2 \pi / 3, R_{0}=1.5$. 


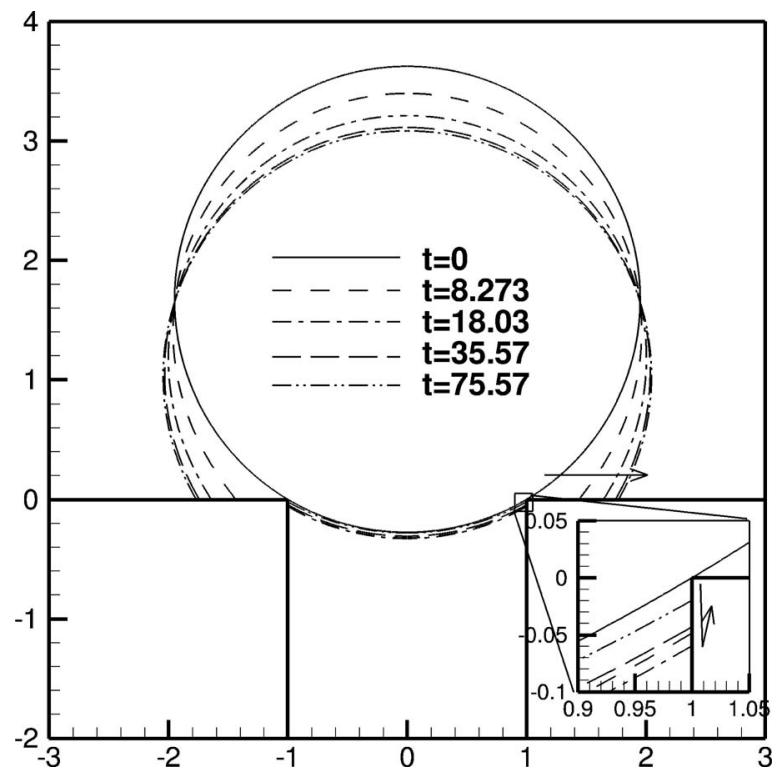

FIG. 9. Drop penetration with $\theta_{S} \in(\pi / 2,3 \pi / 4)$ and $R_{0} \in\left(R_{c}, R_{p}\right) . \theta_{S}=2 \pi / 3, R_{0}=1$.95. The inset shows a close view at the corner.

shrinks, the penetration rate increases with time between $t=798.9$ and 2363 , which is borne out by the decreasing time intervals and increasing $l$ increments between interface snapshots in Fig. 11(b). The same conclusion can also be made from the $l \sim t$ curve in Fig. 18(b). Eventually, the drop completely penetrates the pore (after a long time).

The exact drop radius $R_{c}^{*} \in\left(R_{c}, R_{p}\right)$ that differentiates penetration and non-penetration relies on the initial transient while the drop is still spreading. In the real systems, this $R_{c}^{*}$ may depend on inertia, slip length, contact angle hysteresis, etc., which are out of the scope of this paper. Even in our simulations, such near critical calculations take extremely long times, therefore we do not intend to find the exact $R_{c}^{*}$ here. However, the following simple analysis shows that $R_{c}^{*}$ should be only slightly larger than $R_{c}$. In Regime III (cf. Fig. 4), as $R_{0}$ is closer to $R_{p}$ than to $R_{s}$, we know that the drop is easier to spread on the outer solid surface than to penetrate into the pore. More support can be obtained from the driving force of the contact line, i.e., the unbalanced Young's force, as shown in Fig. 12. It can be seen that the unbalanced Young's force is much smaller at contact line A than at contact line B. Therefore, the drop spreads much faster than it penetrates, at least initially. This is also consistent with the fact that the time scale for drop spreading $\left(<10^{2}\right)$ is much shorter
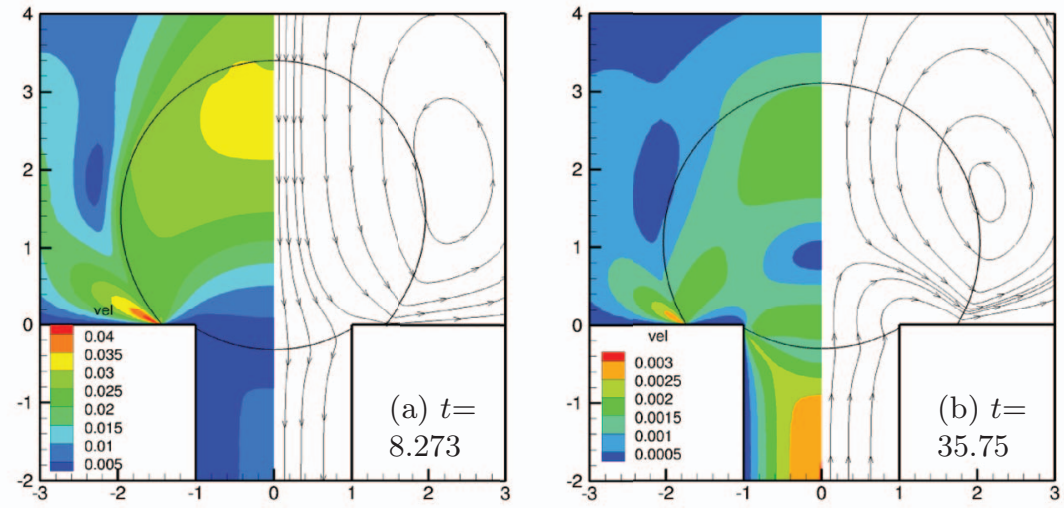

FIG. 10. Contours of velocity magnitude $|\boldsymbol{v}|$ and streamlines. $\theta_{S}=2 \pi / 3, R_{0}=1.95$. 

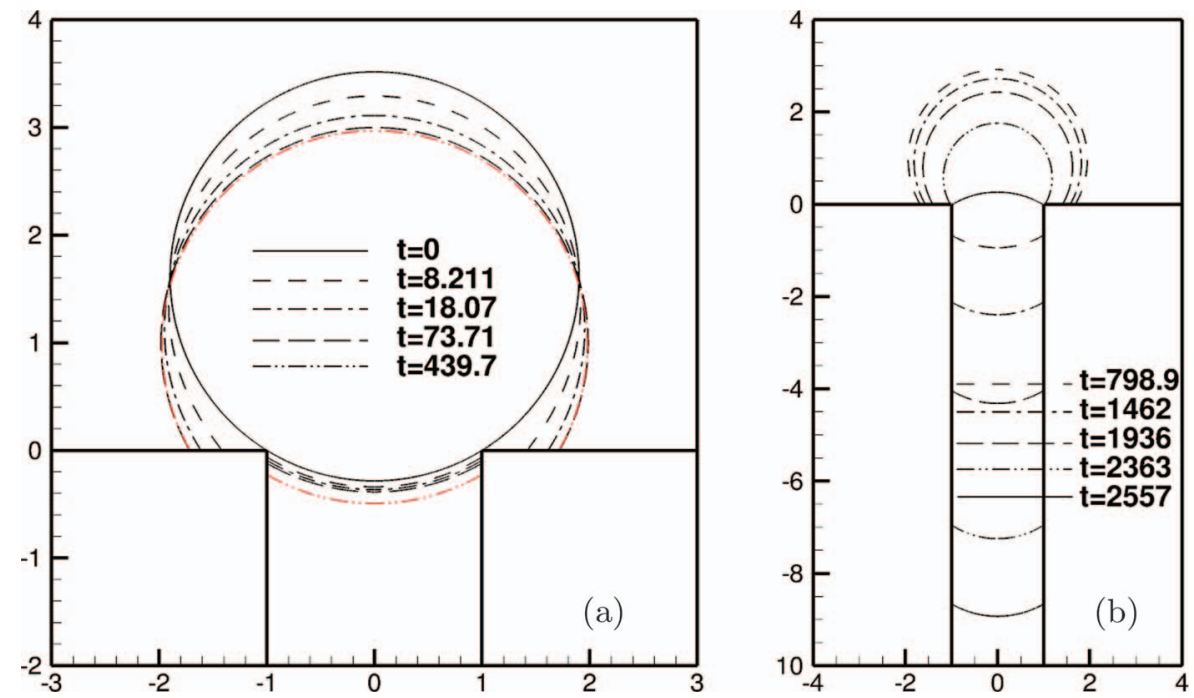

FIG. 11. (a, b) Drop penetration with $\theta_{S} \in(\pi / 2,3 \pi / 4)$ and $R_{0}=R_{C} . \theta_{S}=2 \pi / 3$.

than that for penetration $\left(>10^{3}\right)$ in Fig. 11. This separation of time scales is expected to be more pronounced as $R_{0}$ increases. Therefore, it is possible for the initial transient to pass $l_{c}$ only when $l_{c}$ is small enough, i.e., when $R_{0}$ is very close to $R_{c}$. Even if the drop penetrates the pore, it takes a long time, at least longer than that with $R_{0}=R_{c}$. To conclude, penetration in Regime III only occurs when $R_{0}$ is very close to $R_{c}$ and it takes a long time to complete.

\section{Regime IV, $R_{0} \in\left(R_{p}, \infty\right)$}

We choose $R_{0}=2.5$ as an example and the result is shown in Fig. 13. At $t=0, \theta_{1}=113.6^{\circ}$ $<\theta_{S}$, and $\theta_{2}=156.4^{\circ}>\theta_{S}$. Thus, the outer portion of the drop spreads on the outer solid surface while the meniscus stays pinned at the pore inlet. Eventually, the drop stays at the pore inlet with no penetration $(l=0)$. At $t=171.3$, the drop surface has $R_{1}=2.639$ and $R_{2}=2.637$, and the system is almost in static equilibrium.

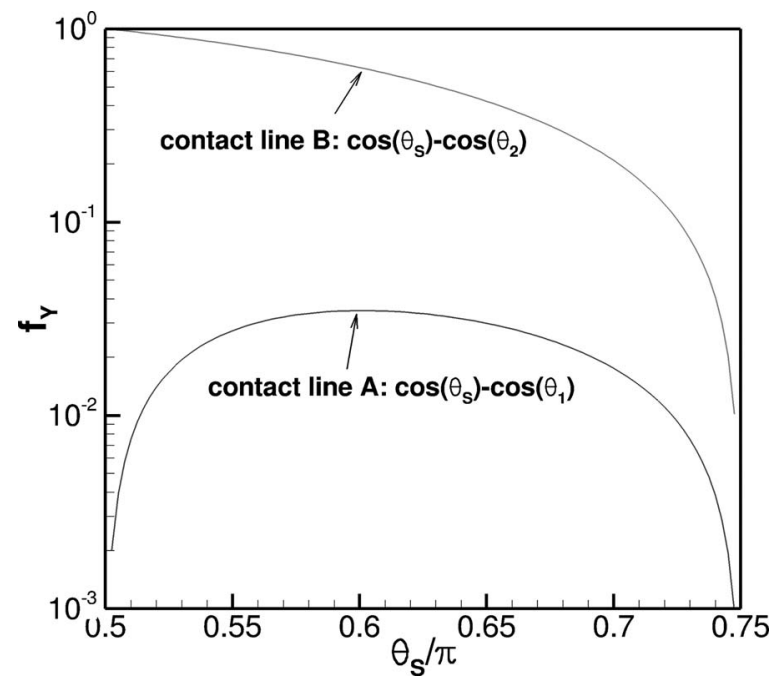

FIG. 12. Unbalanced Young's force at $t=0$ for $R_{0}=R_{c}$. 


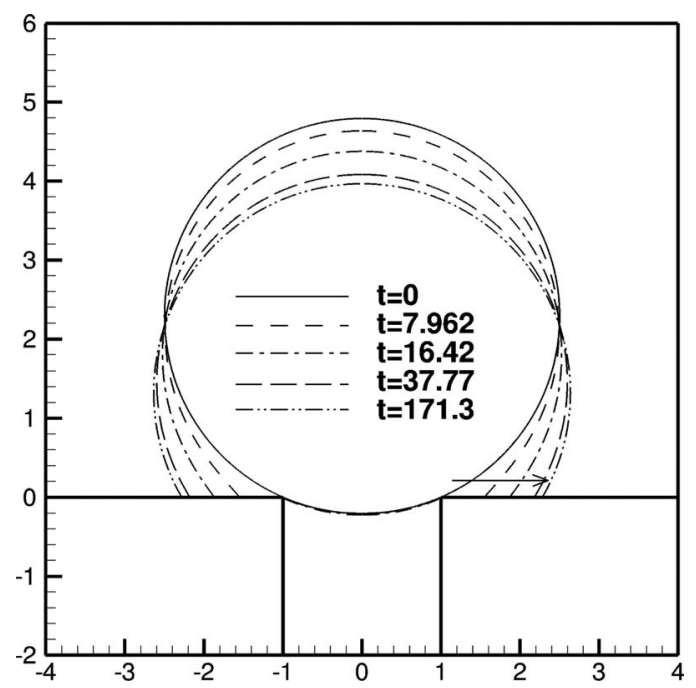

FIG. 13. Drop penetration with $\theta_{S} \in(\pi / 2,3 \pi / 4)$ and $R_{0}>R_{p} . \theta_{S}=2 \pi / 3, R_{0}=2.5$.

\section{B. $\theta_{s} \in(3 \pi / 4, \pi)$}

Only three regimes are involved for this $\theta_{S}$ range. We take $\theta_{S}=5 \pi / 6$ to do the simulations, which has $R_{p}=\frac{2}{\sqrt{3}} r_{0}=1.1547 r_{0}$ and $R_{s}=2 r_{0}$. In the following, we lower the initial drop position a little bit so that the $\phi=0.89$ contour, with a distance $2 \epsilon=0.02$ from the $\phi=0$ contour, goes through the corner point (cf. Fig. 15(b)). This allows more contact between the diffuse interface and the solid surface; otherwise, the high hydrophobicity may undesirably expel the drop from the solid surface.

- Regime I, $R_{0} \in\left(r_{0}, R_{p}\right)$. Figure 14 shows the results with $R_{0}=1$.1. The penetration process is almost the same as that in Fig. 5. Note that the penetration in the current case is much slower because $R_{0}$ is quite close to $R_{p}$.

- Regime $\mathrm{V}, R_{0} \in\left(R_{p}, R_{s}\right)$. This regime is only unique to $\theta_{S}>3 \pi / 4$. When the drop touches the pore, we have $\theta_{1}=131.8^{\circ}<\theta_{S}$ and $\theta_{2}=138.2^{\circ}<\theta_{S}$. As a result, contact lines do not spread out in either direction and the drop remains spherical. A typical case with $R_{0}=1.5$ is shown in Fig. 15. Initially, most of the diffuse interface is in contact with the solid surface at the corner. The high hydrophobicity drives the drop $(\phi=1)$ away from the solid surface. At $t=25.86$, the diffuse interface is almost completely separated from the solid surface.

- Regime IV, $R_{0} \in\left(R_{s}, \infty\right)$. Figure 16 shows the result with $R_{0}=2.5$. Similar to Fig. 13, the drop spreads on the outer solid surface without any penetration into the pore.

\section{ENERGY BARRIER}

In this section, we study the evolution of surface energy of the whole system when a drop enters the pore. If the surface energy keeps decreasing during the whole process, then the drop enters the pore spontaneously. Otherwise, an external force is required to overcome the increasing surface energy.

For simplicity, we start from a state in which contact line $A$ is pinned at the corner, and $B$ spreads out (if it tends to spread), and $R_{1}=R_{p}$. Note that this starting state (cf. $l=0$ in Fig. 3(c) or 3(d)) is not necessarily in equilibrium. Then, the drop enters the pore either spontaneously or by external force, until complete penetration as shown in Fig. 3(e) is achieved. The penetration process considered here has two stages. In the first stage (cf. Figs. 3(b)-3(d)), contact line $B$ retracts to the corner with $\theta_{2}=\theta_{S}$; in the second stage (cf. Figs. 3(d) and 3(e)), $\theta_{2}$ decreases from $\theta_{S}$ to $\theta_{S}-\pi / 2$ 


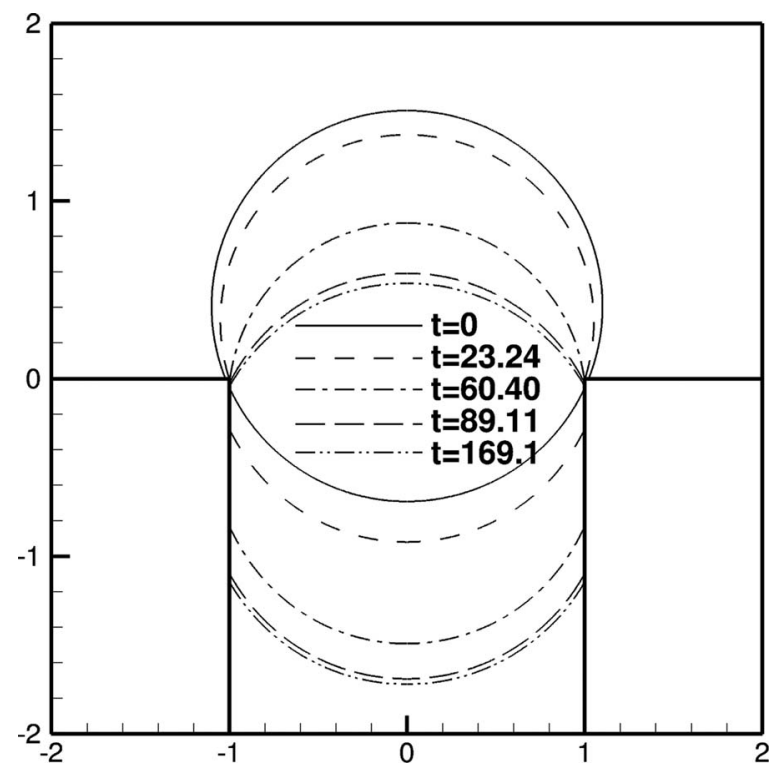

FIG. 14. Drop penetration with $\theta_{S} \in(3 \pi / 4, \pi)$ and $R_{0} \in\left(r_{0}, R_{p}\right) . \theta_{S}=5 \pi / 6, R_{0}=1.1 r_{0}$.

with contact line $B$ pinned at the corner. Note that for a small drop that does not spread on the outer solid surface, the first stage is absent.

To make the analysis manageable, we further assume that all drop surfaces are spherical and the contact angle is always $\theta_{S}$ except at the corner $C$. Then, the surface energy relative to the initial state (Fig. 3(a)) is

$$
E=\left(S_{1}+S_{2}\right) \sigma+S_{3}\left(-\cos \theta_{S}\right) \sigma-S_{0} \sigma
$$

where $S_{1}$ and $S_{2}$ are the surface areas of the spherical caps inside and outside the pore, $S_{3}$ is the area of the solid surface that is in contact with the liquid drop, and $S_{0}$ is the initial surface area of the drop. In the general case as shown in Fig. 3(c), these areas are

$$
\begin{aligned}
& S_{1}=2 \pi R_{1} h_{1}, \\
& S_{2}=2 \pi R_{2} h_{2},
\end{aligned}
$$
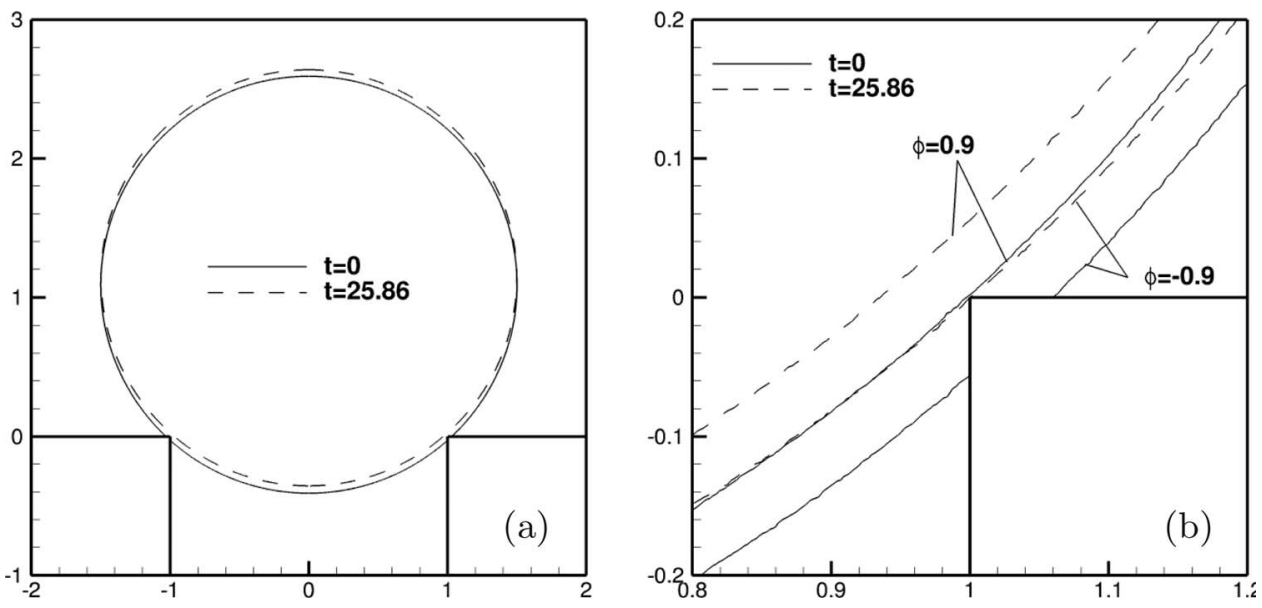

FIG. 15. (a) Drop penetration with $\theta_{S} \in(3 \pi / 4, \pi)$ and $R_{0} \in\left(R_{p}, R_{S}\right) . \theta_{S}=5 \pi / 6, R_{0}=1.5 r_{0}$. (b) The magnified view of $\phi$ $= \pm 0.9$ contours at the corner. 


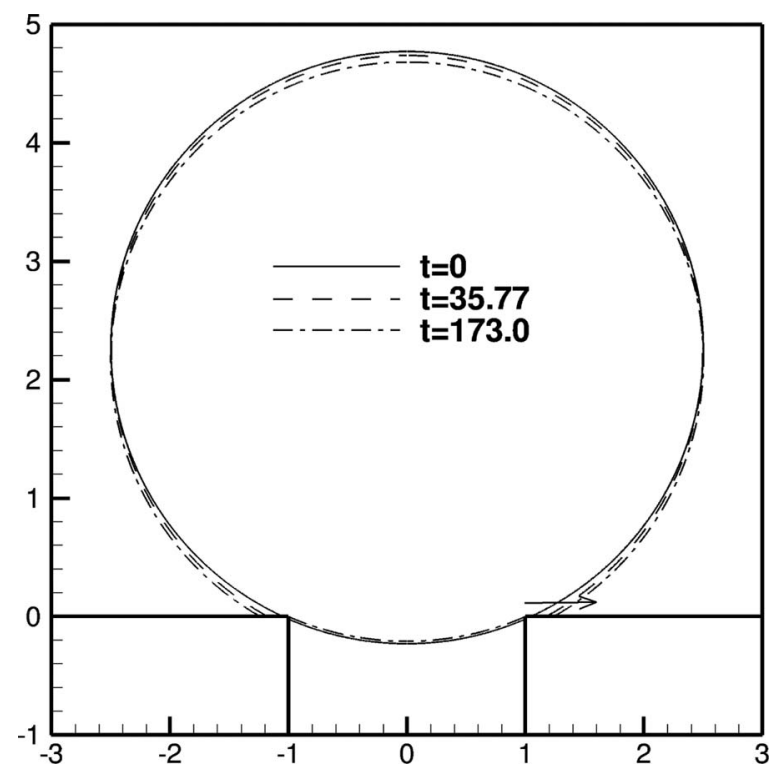

FIG. 16. Drop penetration with $\theta_{S} \in(3 \pi / 4, \pi)$ and $R_{0}>R_{S} . \theta_{S}=5 \pi / 6, R_{0}=2.5$.

and

$$
S_{3}=\pi\left(r_{2}^{2}-r_{0}^{2}\right)+2 \pi r_{0} l,
$$

respectively. Here, $R_{1}=r_{0} /\left(-\cos \theta_{S}\right)$ and $h_{1}=R_{1}\left(1-\sin \theta_{S}\right)=r_{0}\left(1-\sin \theta_{S}\right) /\left(-\cos \theta_{S}\right)$ are only dependent on $r_{0}$ and $\theta_{S}$. Therefore $S_{1}$ is constant during each individual penetration process. In stage one, we have $R_{2}=h_{2} /\left(1-\cos \theta_{S}\right)$ and $r_{2}=h_{2} \sin \theta_{S} /\left(1-\cos \theta_{S}\right)$ (cf. Fig. 3(c)). In stage two, we have $R_{2}=\frac{r_{0}^{2}+h_{2}^{2}}{2 h_{2}}$ and $r_{2}=r_{0}$ (cf. Fig. 3(d)). Note that stage one requires $r_{2} \geq r_{0}$, i.e., $\frac{h_{2}}{r_{0}} \geq \frac{1-\cos \theta_{S}}{\sin \theta_{S}}$. For a given drop, $\theta_{S}$ and $R_{0}$ are both fixed, so the surface energy can be expressed as a function of $h_{2}$ and $l$.

The relation between $h_{2}$ and $l$ can be uniquely determined by the volume conservation

$$
V_{0}=V_{1}+V_{2}+V_{3},
$$

where

$$
V_{0}=\frac{4}{3} \pi R_{0}^{3}
$$

is the initial drop volume,

$$
V_{3}=\pi r_{0}^{2} l
$$

is the volume of the liquid cylinder inside the pore, and $V_{1}$ and $V_{2}$ are the volumes of the spherical caps given by Eqs. (17) and (18). As $R_{1}$ and $h_{1}$ only depends on $\theta_{S}$ and $r_{0}$, so does $V_{1}$. Equation (24) gives

$$
h_{2}=\left\{\frac{V_{0}-V_{1}-\pi r_{0}^{2} l}{\frac{\pi}{6}\left[3\left(\frac{\sin \theta_{S}}{1-\cos \theta_{S}}\right)^{2}+1\right]}\right\}^{1 / 3}, \text { for } l \in\left[0, l_{b}\right]
$$

in the first stage and

$$
\frac{\pi}{6} h_{2}\left(3 r_{0}^{2}+h_{2}^{2}\right)=V_{0}-V_{1}-\pi r_{0}^{2} l, \text { for } l \in\left[l_{b}, l_{\max }\right]
$$


in the second stage. Here

$$
l_{b}=\frac{V_{0}-V_{1}-\frac{\pi}{6} r_{0}^{3}\left[3+\left(\frac{1-\cos \theta_{S}}{\sin \theta_{S}}\right)^{2}\right]\left(\frac{1-\cos \theta_{S}}{\sin \theta_{S}}\right)}{\pi r_{0}^{2}}
$$

is the boundary between the two stages, which can be obtained from Eq. (27) or Eq. (28) by letting $h_{2}=\frac{r_{0}\left(1-\cos \theta_{s}\right)}{\sin \theta_{S}}$. A negative $l_{b}$ means that the initial drop is too small and does not spread on the outer solid surface, i.e., the first stage is missing. $l_{\max }$ is the maximal penetration depth

$$
l_{\max }=\frac{V_{0}-2 V_{1}}{\pi r_{0}^{2}} .
$$

Equation (28) is a cubic equation for $h_{2}$, which has only one real solution

$$
h_{2}=\frac{-2 \cdot 3^{1 / 3} a+2^{1 / 3} b^{2 / 3}}{6^{2 / 3} b^{1 / 3}}
$$

where $a=3 r_{0}^{2}, b=9 c+\sqrt{12 a^{3}+81 c^{2}}, c=\frac{V_{0}-V_{1}-\pi r_{0}^{2} l}{\pi / 6}$. Eventually, given $\theta_{S}, R_{0}$, and $r_{0}$, the surface energy can be expressed as a function of only one variable $l$ by combining Eqs. (20)-(23), (25), (17), (27), and (31).

The curves of $E(l)$ for different $\theta_{S}$ and $R_{0}$ are shown in Fig. 17. All the curves terminate at $l=l_{\max }$, which corresponds to the complete penetration as shown in Fig. 3(e). $E(l)$ decreases monotonically when $R_{0} \leq R_{c}$ (if $\theta_{S}<3 \pi / 4$, cf. Fig. 17(a)) or $R_{0} \leq R_{p}$ (if $\theta_{S}>3 \pi / 4$, cf. Fig. 17(c)). Consequently, the drop enters the pore spontaneously, which is consistent with Sec. III. For other curves in Fig. 17, an energy barrier is formed due to a local maximum. The drop is not able to enter the pore unless this energy barrier can be overcome by factors such as external force, inertia, ${ }^{34}$ and thermal fluctuations at molecular scales. ${ }^{19}$ It is interesting to note that the maximum of the $R_{0}=R_{p}$ curve in Fig. 17(a) is lower than zero, which is the initial energy of the system. This means that the system may still get over the energy barrier depending on the initial transient. According to the phase-field simulations, for $\theta_{S}=2 \pi / 3$, drops with initial radii $R_{0}=1.95<R_{p}$ still cannot overcome the energy barrier (cf. Fig. 9). However, the height of energy barrier $\left(E\left(l_{c}\right)-E(0)\right)$ approaches zero as $R_{0} \rightarrow R_{c}$, therefore we do expect the initial transient to overcome the energy barrier when $R_{0}$ is close enough to $R_{c}$.

The critical $l\left(\right.$ or $\left.h_{2}\right)$ at which the system is in equilibrium can be obtained from $\frac{d E}{d l}=0$ (or $\left.\frac{d E}{d h_{2}}\right)$. From $\frac{d E}{d h_{2}}=0$, we find the following critical points:

$$
h_{c 1}=\frac{1-\cos \theta_{S}}{-\cos \theta_{S}} r_{0}
$$

in the first stage and

$$
h_{c 2}=\frac{1+\sin \theta_{S}}{-\cos \theta_{S}} r_{0}, h_{c 3}=\frac{1-\sin \theta_{S}}{-\cos \theta_{S}} r_{0}
$$

in the second stage. If we further take into account the requirement $h_{2} / r_{0} \geq\left(1-\cos \theta_{S}\right) / \sin \theta_{S}$ for the first stage and otherwise for the second, then we can see that $h_{c 1}$ is only valid for $\theta_{S} \leq 3 \pi / 4, h_{c 2}$ is valid for $\theta_{S} \geq 3 \pi / 4$, and $h_{c 3}$ is valid for the whole non-wetting range $\theta_{S} \in(-\pi / 2, \pi)$. The critical length $h_{2}$ can be easily translated into critical penetration depth $l$. $h_{c 3}$ corresponds to the maximum penetration $l_{\max }$, while $h_{c 1}$ and $h_{c 2}$ correspond to the local maxima of the surface energy curves in Fig. 17:

$$
l_{c}=\frac{4\left(R_{0}^{3}-R_{c}^{3}\right)}{3 r_{0}^{2}}, \text { if } \theta_{S} \in(\pi / 2,3 \pi / 4]
$$

or

$$
l_{c}=\frac{4\left(R_{0}^{3}-R_{p}^{3}\right)}{3 r_{0}^{2}}, \text { if } \theta_{S} \in[3 \pi / 4, \pi) .
$$



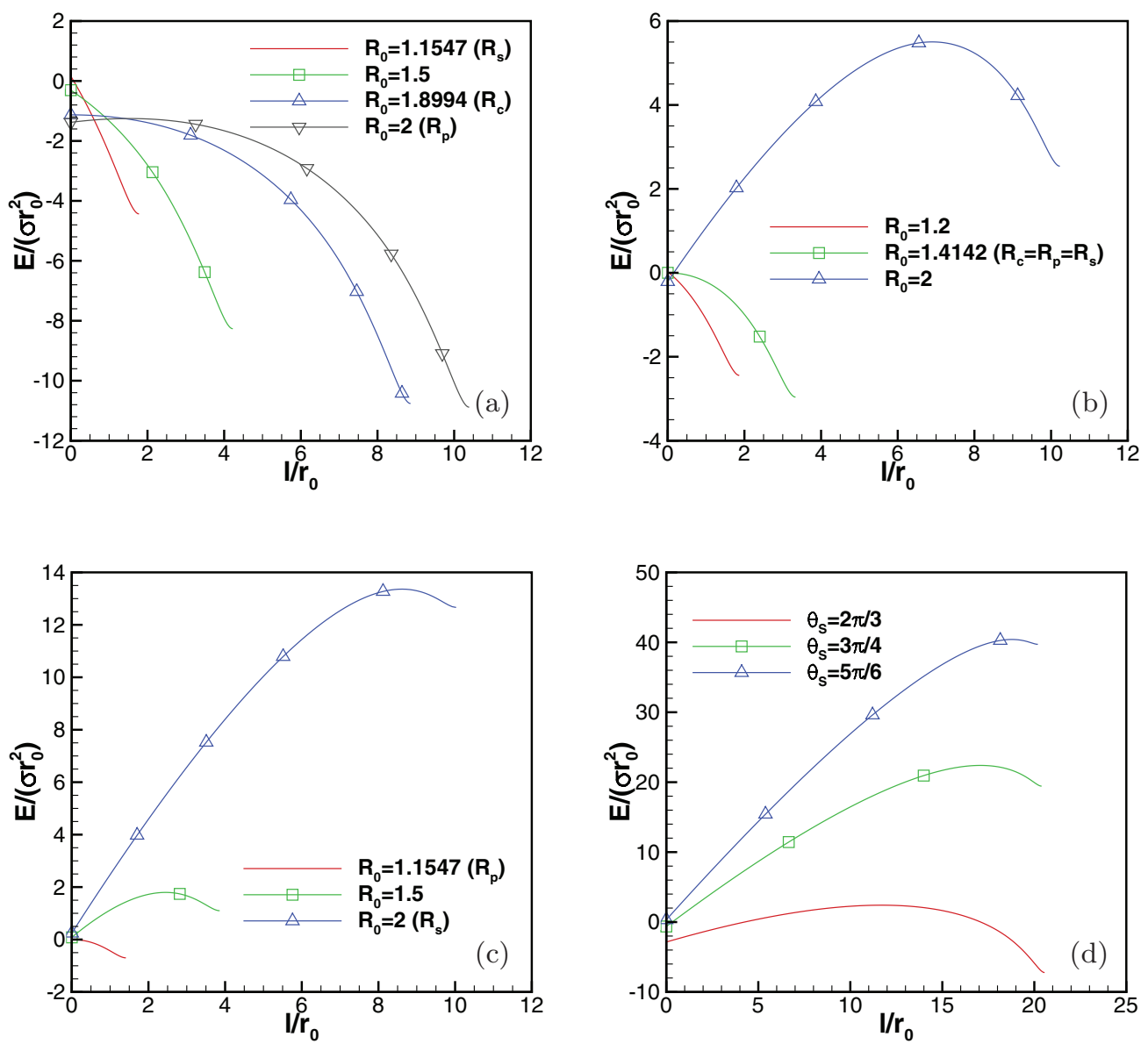

FIG. 17. Surface energy as a function of penetration depth. (a) $\theta_{S}=2 \pi / 3$; (b) $\theta_{S}=3 \pi / 4$; (c) $\theta_{S}=5 \pi / 6$; (d) $R_{0}=2.5 r_{0}$.

It is obvious that $l_{\max }$ (cf. Fig. 3(d)) is stable while $l_{c}$ (cf. Fig. 3(c) for $\theta_{S} \leq 3 \pi / 4$ and Fig. 3(d) for $\theta_{S} \geq 3 \pi / 4$ ) is unstable. At the critical drop volume, the local maximum moves to $l_{c}=0$, as shown by the $R_{0}=R_{c}$ curves in Figs. 17(a) and 17(b) and the $R_{0}=R_{p}$ curve in Fig. 17(c). Note that the partial penetration as shown in Figs. 3(c) and 3(d) is still possible if gravity is considered or if the pore has a varying cross-sectional area. ${ }^{35}$

The energy barrier can also be interpreted utilizing the critical drop volume. If the drop volume is larger than the critical volume for spontaneous penetration, then an external force is needed to push the extra volume $\pi r_{0}^{2} l_{c}$ into the pore, after which the surface energy of the system passes the local maximum and the drop penetrates the pore spontaneously. A relevant practical problem may be that of the waterproof breathable cloth. Tiny rain drops that originally could not get into the cloth may be pressed in by strong winds.

\section{DYNAMICS OF PENETRATION}

The Lucas-Washburn (LW) equation, ever since its development by Lucas ${ }^{5}$ and Washburn, ${ }^{6}$ has been the basis for the analysis of capillary penetration. This equation considers a fully developed Poiseuille flow, where the capillary force is balanced by the viscous drag and possibly the gravitational force. Its notably simple solution predicts that $l \propto \sqrt{t}$, where $l$ is the penetration depth and $t$ is the time with $t=0$ at the onset of penetration. Shortly after the work of Lucas, ${ }^{5}$ Washburn, ${ }^{6}$ Rideal, ${ }^{36}$ and Bosanquet ${ }^{37}$ took inertia into consideration, which avoids the burst-like behavior at $t$ $=0$ in the original LW equation. In the last century, various modifications have been done on the 

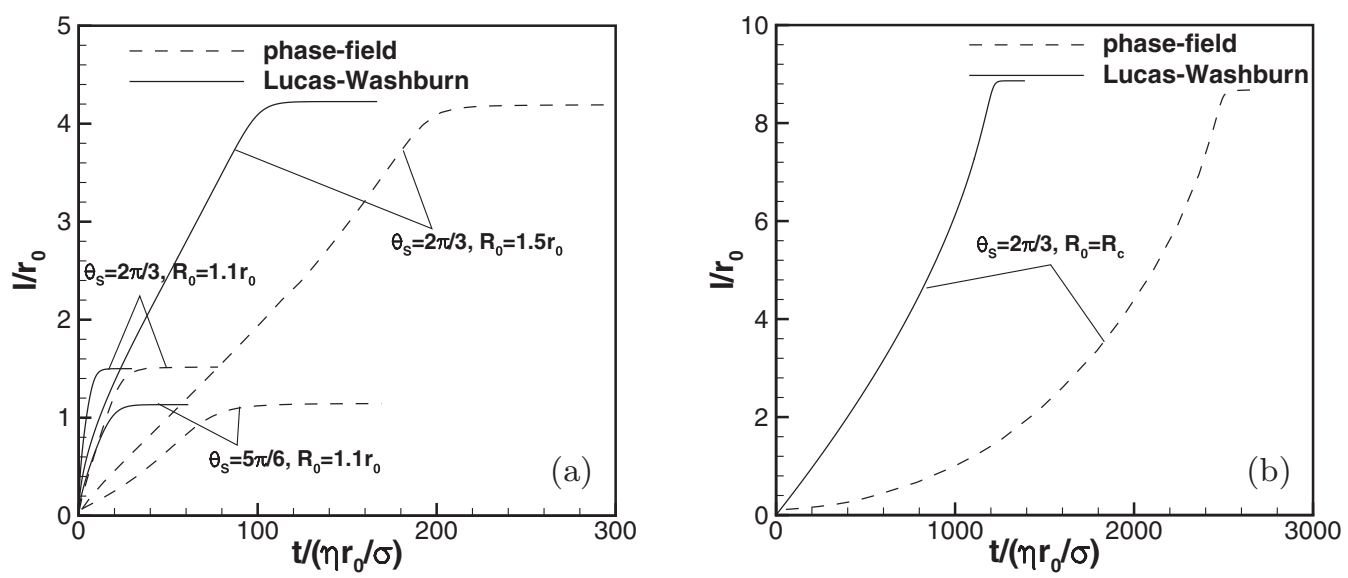

FIG. 18. (a, b) Transient of drop penetration.

LW equation to account for different effects including tube edge and contact line. A short summary on these early modifications can be found in Ref. 38 .

For the penetration of finite-size drops, following the work of Marmur, ${ }^{14,39}$ we modify the LW equation as follows:

$$
8 \pi \eta l \frac{d l}{d t}=\pi r_{0}^{2}\left(\frac{2}{R_{2}}+\frac{2 \cos \theta_{S}}{r_{0}}\right) \sigma
$$

The left-hand side is the drag force of fully developed Poiseuille flow with length $l$ and average velocity $\frac{d l}{d t}$. The right-hand side can be obtained from the derivative of the surface energy $-\frac{d E(l)}{d l}$, or equivalently from the difference of the Laplace pressures generated by the two spherical caps with radii $R_{2}$ and $R_{1}=\frac{r_{0}}{-\cos \theta_{S}}$. $R_{2}$ depends on $h_{2}$ and can be consequently expressed as a function of $l$. Eventually, Eq. (36) can be written as an ordinary differential equation for $l^{2}$. Note that this equation recovers the original LW equation if $R_{2}$ approaches infinity.

The modified LW equation (36) with initial condition $l(0)=0$ can be solved numerically using the ordinary differential equation (ODE) solvers in MATLAB. A comparison with phase-field simulations is shown in Fig. 18. For all the cases, the LW equation greatly overestimates the penetration rate $d l / d t$. This is expected as Eq. (36) only considers the dissipation from the Poiseuille flow inside the pore, while the actual flow has dissipation from many other sources, e.g., flow in the protruding drop, flow at the pore entrance, and moving contact lines.

In viscous flow, the viscous dissipation rate per unit volume is given by

$$
\psi=\tau: \nabla \boldsymbol{v}
$$

A typical distribution of $\psi$ in phase-field simulations is shown in Fig. 19(a). First of all, there is non-negligible dissipation in the drop portion outside the pore. Second, the dissipation near the ends of the liquid slug deviates from that for the fully developed Poiseuille flow. Finally, there is strong dissipation constrained in a small region near the contact line. This part of the dissipation reflects the effect of moving contact line: $\sigma\left(\cos \theta_{S}-\cos \theta_{A}\right) \frac{d l}{d t}$. Note that the Cahn-Hilliard equation has built-in dissipation, with a rate density $\gamma|\nabla G|^{2}{ }^{30}$ In the current computations, this dissipation is much smaller than the viscous one and is therefore not included in the following discussions. The viscous dissipation rate can be obtained by the integration

$$
\Phi_{P F}=2 \pi \int_{\Omega} \psi x d \Omega
$$



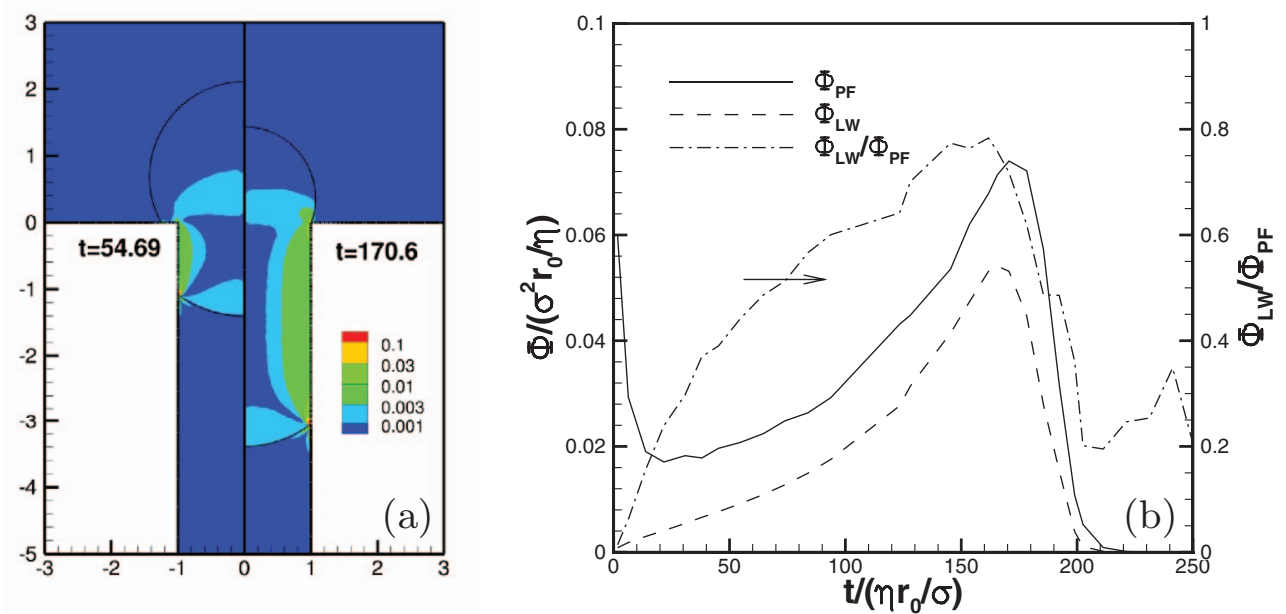

FIG. 19. Viscous dissipation for $\theta_{S}=2 \pi / 3$ and $R_{0}=1.5 r_{0}$. (a) The dissipation rate per unit volume in the phase-field simulations. The left and right halves are from two different time instants. (b) The viscous dissipation rate versus time.

where $x$ is the coordinate in the radial direction and $\Omega$ is the two-dimensional computational domain. In the LW equation, the dissipation rate is found to be

$$
\Phi_{L W}=8 \pi \eta l\left(\frac{d l}{d t}\right)^{2}
$$

which is the product of drag force and penetration rate. The comparison of the dissipation from these two methods is shown in Fig. 19(b). Here, $l$ and $\frac{d l}{d t}$ used in $\Phi_{L W}$ are measured from the phase-field simulations. At $t=0$, the microscopic contact angles immediately change from $\theta_{1}$ and $\theta_{2}$ to $\theta_{S}$, which causes a severe attenuation of the interface and a high viscous stress at the contact lines. As a result, $\Phi_{P F}$ starts at a non-zero value, which is reflected in $\Phi_{L W}$. As the interface relaxes, the dissipation rate quickly drops and minimizes around $t=20$. The subsequent increase in dissipation rate, both $\Phi_{P F}$ and $\Phi_{L W}$, is a result of the increasing penetration depth and rate. At the same time, the dissipation from the Poiseuille flow begins to catch up. The ratio $\Phi_{L W} / \Phi_{P F}$ gives a rough idea on how accurately the LW equation can predict the penetration dynamics. We expect the LW equation to recover the exact penetration dynamics when $\Phi_{L W} / \Phi_{P F}=1$. The small ratio at the early stage means that only a small portion of the dissipation is accounted for in the LW equation, and therefore the rate of penetration is greatly overpredicted. As $l$ increases, the dissipation from the Poiseuille flow begins to dominate. At $t=150, l \approx 3$, and $\Phi_{L W} / \Phi_{P F} \approx 80 \%$. For the calculation with $R_{0}=R_{c}$ and $\theta_{S}=2 \pi / 3$, the ratio $\Phi_{L W} / \Phi_{P F}$ can achieve $90 \%$ when $l \approx 8$. This ratio is expected to approach 1 as $l$ further increases. However, for drop penetration, the penetration depth is limited by the critical drop volume. Therefore, generally, $\Phi_{L W} / \Phi_{P F}$ is too low for the $L W$ equation to reasonably predict the penetration dynamics of drops. This is different from the liquid penetration from an infinite reservoir, ${ }^{27}$ in which the penetration depth can be very large.

It should be noted that the final penetration depths in the phase-field simulations are slightly lower than the theoretical values, especially when the computational time is long. For example, at $\theta_{S}$ $=120^{\circ}$, the final depths in the phase-field simulations are $l=4.192$ and $l=8.676$ for $R_{0}=1.5$ and $R_{0}=R_{c}$, respectively, while the theoretical values are $l_{\max }=4.226$ and 8.862. This slight shrinkage of drop volume is inherent in the phase-field method, which may become noticeable for long-time simulations. ${ }^{40}$

\section{CONCLUSIONS}

It has been previously shown that a non-wetting drop may still penetrate a capillary tube spontaneously, due to the capillary pressure inside drop. The current work proposes a drop penetration 
model which is more realistic for porous media. The capillary tube is replaced by an exposed pore, where the drop spreading on the outer surface can be accounted for. We tackle this problem both theoretically and numerically. To make the problem manageable, we neglect inertia and gravity. The major conclusions are as follows:

(a) The spontaneous penetration of a non-wetting drop can be divided into 5 regimes based on the static contact angle $\theta_{S}$ and the initial drop radius $R_{0}$ : (I) penetration without spreading, (II) penetration with spreading, (III) penetration with spreading that relies on the initial transient, (IV) non-penetration with spreading, and (V) non-penetration without spreading. These regimes are delineated by critical radii $R_{p}=r_{0} /\left(-\cos \theta_{S}\right), R_{s}=r_{0} / \sin \theta_{S}$, and $R_{c}$ given by Eq. (19).

(b) For $\theta_{S} \in(\pi / 2,3 \pi / 4)$, i.e., weakly non-wetting drops, spontaneous drop penetration is guaranteed only if $R_{0}<R_{c}$, which is smaller than $R_{p}$ for capillary tubes because of drop spreading. For $\theta_{S} \in(3 \pi / 4, \pi)$, i.e., strongly non-wetting drops, the critical drop radius is $R_{p}$, the same as that for capillary tubes.

(c) The evolution of surface energy reveals a local maximum for the drops of size larger than $R_{c}$ for $\theta_{S} \in(\pi / 2,3 \pi / 4]$ or $R_{p}$ for $\theta_{S} \in[3 \pi / 4, \pi)$, which constitutes an energy barrier for penetration. The partial penetration with mechanical equilibrium is unstable. In Regime III, this energy barrier may be overcome by the initial transient if it is very low. Otherwise, external forces are needed to overcome this barrier.

(d) The Lucas-Washburn equation greatly overpredicts the penetration rate, because it only considers the dissipation of the fully developed Poiseuille flow, which may be only a small portion of the total dissipation. This deficiency is especially prominent when the penetration depth is small due to the finite drop size.

All of our theoretical analyses are supported by our phase-field simulations. However, we have to point out that our model is still simplified in that we neglect inertia, gravity, and more importantly, contact angle hysteresis. The inertia and the gravity may be negligible at small length scales. But the contact angle hysteresis always exists as long as the length scale of the problem is above that of the surface roughness or chemical heterogeneity.

\section{ACKNOWLEDGMENTS}

This research is supported by NSF-DMS (Grant No. 0907788). Part of the work was done when P. Yue was visiting Tiezheng Qian at the Hong Kong University of Science and Technology. We thank James J. Feng (University of British Columbia) and Howard A. Stone (Princeton University) for helpful discussions that encouraged us to look at this problem.

${ }^{1}$ N. R. Morrow and G. Mason, "Recovery of oil by spontaneous imbibition," Curr. Opin. Colloid Interface Sci. 6, 321-337 (2001).

2 I. Pezron, G. Bourgain, and D. Quéré, "Imbibition of a fabric,” J. Colloid Interface Sci. 173, 319-327 (1995).

${ }^{3}$ J. Kettle, T. Lamminmäki, and P. Gane, "A review of modified surfaces for high speed inkjet coating," Surf. Coat. Technol. 204, 2103-2109 (2010).

${ }^{4}$ U. Pasaogullari and C.-Y. Wang, "Two-phase transport and the role of micro-porous layer in polymer electrolyte fuel cells," Electrochim. Acta 49, 4359-4369 (2004).

${ }^{5}$ R. Lucas, "Ueber das zeitgesetz des kapillaren aufstiegs von flussigkeiten," Kolloid-Z. 23, 15 (1918).

${ }^{6}$ E. W. Washburn, "The dynamics of capillary flow," Phys. Rev. 17, 273-283 (1921).

${ }^{7}$ D. Quéré, "Inertial capillarity," Europhys. Lett. 39, 533-538 (1997).

${ }^{8}$ M. Radiom, W. K. Chan, and C. Yang, "A study of capillary flow from a pendant droplet," Microfluid. Nanofluid. 7, 697-707 (2009)

${ }^{9}$ A. V. Bazilevsky, K. G. Kornev, A. N. Rozhkov, and A. V. Neimark, "Spontaneous absorption of viscous and viscoelastic fluids by capillaries and porous substrates," J. Colloid Interface Sci. 262, 16-24 (2003).

${ }^{10}$ M. Reyssat, L. Courbin, E. Reyssat, and H. A. Stone, "Imbibition in geometries with axial variations," J. Fluid Mech. 615, 335-344 (2008).

${ }^{11}$ B. V. Zhmud, F. Tiberg, and K. Hallstensson, "Dynamics of capillary rise," J. Colloid Interface Sci. 228, 263-269 (2000).

${ }^{12}$ K. G. Kornev and A. V. Neimark, "Spontaneous penetration of liquids into capillarie and porous membranes revisited," J. Colloid Interface Sci. 235, 101-113 (2001).

${ }^{13}$ P. Joos, P. van Remoortere, and M. Bracke, “The kinetics of wetting in a capillary,” J. Colloid Interface Sci. 136, 189-197 (1990). 
${ }^{14}$ A. Marmur, "Penetration of a small drop into a capillary," J. Colloid Interface Sci. 122, 209-219 (1988).

${ }^{15}$ A. Marmur, "The radial capillary," J. Colloid Interface Sci. 124, 301-308 (1988).

${ }^{16}$ M. Denesuk, G. L. Smith, B. J. J. Zelinski, N. J. Kreidl, and D. R. Uhlmann, "Capillary penetration of liquid droplets into porous materials," J. Colloid Interface Sci. 158, 114-120 (1993).

${ }^{17}$ K. P. Hapgood, J. D. Litster, S. R. Biggs, and T. Howes, "Drop penetration into porous powder beds," J. Colloid Interface Sci. 253, 353-366 (2002).

${ }^{18}$ S. Bekou and D. Mattia, "Wetting of nanotubes," Curr. Opin. Colloid Interface Sci. 16, 259-265 (2011).

${ }^{19}$ D. Schebarchov and S. C. Hendy, "Uptake and withdrawal of droplets from carbon nanotubes," Nanoscale 3, 134-141 (2011).

${ }^{20}$ M. Moseler, F. Cervantes-Sodi, S. Hofmann, G. Csanyi, and A. C. Ferrai, "Dynamic catalyst restructuring during carbon nanotube growth," ACS Nano 4, 7587-7595 (2010).

${ }^{21}$ D. Schebarchov and S. C. Hendy, "Capillary absorption of metal nanodroplets by single-wall carbon nanotubes," Nano Lett. 8, 2253-2257 (2008).

${ }^{22}$ D. Schebarchov and S. C. Hendy, "Dynamics of capillary absorption of droplets by carbon nanotubes," Phys. Rev. E 78, 046309 (2008).

${ }^{23}$ G. R. Willmott, C. Neto, and S. C. Hendy, "Update of water droplets by non-wetting capillaries," Soft Matter 7, 2357-2363 (2011).

${ }^{24}$ D. Jacqmin, "Contact-line dynamics of a diffuse fluid interface," J. Fluid Mech. 402, 57-88 (2000).

${ }^{25}$ W. Villanueva and G. Amberg, "Some generic capillary-driven flows," Int. J. Multiphase Flow 32, 1072-1086 (2006).

${ }^{26}$ V. V. Khatavkar, P. D. Anderson, and H. E. H. Meijer, "Capillary spreading of a droplet in the partially wetting regime using a diffuse-interface model," J. Fluid Mech. 572, 367-387 (2007).

${ }^{27}$ H. Mehrabian, P. Gao, and J. J. Feng, "Wicking flow through microchannels," Phys. Fluids 23, 122108 (2011).

${ }^{28} \mathrm{P}$. Yue and J. J. Feng, "Wall energy relaxation in the Cahn-Hilliard model for moving contact lines," Phys. Fluids $\mathbf{2 3}$, 012106 (2011).

${ }^{29}$ P. Yue, C. Zhou, and J. J. Feng, "Sharp-interface limit of the Cahn-Hilliard model for moving contact lines," J. Fluid Mech. 645, 279-294 (2010).

${ }^{30} \mathrm{P}$. Yue, C. Zhou, J. J. Feng, C. F. Ollivier-Gooch, and H. H. Hu, "Phase-field simulations of interfacial dynamics in viscoelastic fluids using finite elements with adaptive meshing," J. Comput. Phys. 219, 47-67 (2006).

${ }^{31}$ P. Yue, J. J. Feng, C. Liu, and J. Shen, "A diffuse-interface method for simulating two-phase flows of complex fluids," J. Fluid Mech. 515, 293-317 (2004).

${ }^{32}$ P. Yue and J. J. Feng, "Can diffuse-interface models quantitatively describe moving contact lines?" Eur. Phys. J. Spec. Top. 197, 37-46 (2011).

${ }^{33}$ R. L. Hoffman, "A study of the advancing interface," J. Colloid Interface Sci. 50, 228-241 (1975).

${ }^{34}$ A. Delbos, E. Lorenceau, and O. Pitois, "Forced impregnation of a capillary tube with drop impact," J. Colloid Interface Sci. 341, 171-177 (2010).

${ }^{35}$ T. Ratcliffe and R. H. Davis, "Drop trapping in axisymmetric constrictions with arbitrary contact angle," Phys. Fluids 24, $062102(2012)$

${ }^{36}$ E. K. Rideal, "On the flow of liquids under capillary pressure," Philos. Mag. Ser. 6 44, 1152-1159 (1922).

${ }^{37}$ C. H. Bosanquet, "On the flow of liquids into capillary tubes," Philos. Mag. Ser. 6 45, 525-531 (1923).

${ }^{38}$ A. Marmur, "Penetration and displacement in capillary systems," in Modern Approaches to Wettability: Theory and Applications, edited by M. E. Schrader and G. I. Loeb (Plenum Press, New York, 1992), pp. 327-358.

${ }^{39}$ A. Marmur, "Penetration and displacement in capillary systems of limited size," Adv. Colloid Interface Sci. 39, 13-33 (1992).

${ }^{40}$ P. Yue, C. Zhou, and J. J. Feng, "Spontaneous shrinkage of drops and mass conservation in phase-field simulations," J. Comput. Phys. 223, 1-9 (2007). 